\title{
Mulberry extract to modULate Blood glucosE Responses in noRmoglYcaemic adults (MULBERRY): study protocol for a randomised controlled trial
}

\author{
Mark Lown ${ }^{1 *}$, Richard Fuller ${ }^{1}$, Helen Lightowler ${ }^{2}$, Ann Fraser ${ }^{2}$, Andrew Gallagher ${ }^{3}$, Beth Stuart ${ }^{1}$, \\ Christopher D. Byrne ${ }^{4}$ and George Lewith ${ }^{1}$
}

\begin{abstract}
Background: Worldwide sugar consumption has tripled during the last fifty years. High sugar intake is associated with weight gain and increased incidence of diabetes and has been linked with increased cardiovascular mortality. Reducing the health impact of dietary sugar and poor quality carbohydrate intake is a public health priority. IminoNorm ${ }^{\oplus}$, a proprietary mulberry leaf extract (ME), may reduce blood glucose responses following dietary sugar and carbohydrate intake by reducing absorption of glucose from the gut. Previous research has shown that ME can reduce blood glucose and improve insulin responses in healthy subjects and also in subjects with raised fasting blood glucose levels. Mulberry leaf has an excellent safety profile. This pilot study will test a novel, safe, water soluble product in normoglycaemic adults in the UK to determine if it can reduce glucose absorption without increasing plasma insulin concentration.
\end{abstract}

Methods/design: The trial will be a double-blind, individually randomised, four-arm single-dose crossover design to test the effect of three doses of ME in order to determine efficacy, dose response relationship and gastrointestinal side effects with respect to placebo. A total of 40 subjects will participate in this study and attend for four visits receiving each of the four interventions in random order.

Discussion: We aim to test the evidence that mulberry leaf extract can reduce blood glucose without a disproportionate increase in blood insulin responses in healthy individuals in a high-quality research study based in the UK. It is hoped that this will lead to further randomised controlled trials and an effective dietary supplement to lower blood glucose concentrations.

Trial registration: ISRCTN: ISRCTN14597438 (21 April 2015)

Keywords: Mulberry, Glycaemic response, Insulinaemic response

\section{Background}

Excess calorie intake including those from sugar can make a significant contribution to becoming overweight $[1,2]$ and thus increase the risk of developing type 2 diabetes mellitus (T2DM) [3, 4]. In 2013 a large long-term European study investigating the effect of diet on health [5] found an association between the amount of sugary soft drinks people consumed and their risk of developing type 2 diabetes. In this study weight gain had a large

\footnotetext{
* Correspondence: m.lown@soton.ac.uk

${ }^{1}$ Primary Care \& Population Sciences, Faculty of Medicine, University of Southampton, Aldermoor Health Centre, Southampton SO16 5ST, UK Full list of author information is available at the end of the article
}

effect on diabetes risk, and sugary drinks had a small effect on diabetes risk even after correcting for body mass index (BMI). The global rise in T2DM is linked to the metabolic syndrome (dyslipidemia, hypertension, insulin resistance), and obesity is thought to be one of the greatest risk factors for metabolic syndrome and T2DM [6]. Dietary sugars and carbohydrates play a significant role, as calories from these foods promote fat storage and hunger [7]. A recently completed review of nutrition and its impact on T2DM concluded that dietary restriction of carbohydrate intake is the single most effective approach to manage T2DM [8]. It is estimated that more than 1 in 17 people in the UK have diabetes (diagnosed 
or undiagnosed) [9], and thus reducing the health impact of dietary sugar and poor quality carbohydrate intake is a public health priority. Herbal agents could be quite effective in the management of carbohydrates in the normal diet and in reducing postprandial blood glucose [10].

Mulberry (Morus alba) leaves have been used in traditional Chinese medicine (TCM) for several millennia; its use was first recorded in around $500 \mathrm{AD}$ in the Divine Husbandman's Classic of the Materia Medica [11]. It has also been used for more than 750 years in Japan as an infusion tea [10]. It is used by some human communities for food purposes and in several Asian countries as a herbal tea. Reports have shown that the leaves are nutritious and non-toxic [12]. The Chinese Ministry of Health and the Taiwanese Bureau of Food Safety recognise Morus alba leaves as both a food and a medicine [13]. Mulberry leaf extracts (ME) have a long history of safe use globally for normalising postprandial blood glucose, and it is thought that iminosugars such as 1deoxynojirimycin (DNJ), a reversible, competitive natural $\alpha$-glucosidase inhibitor, are the main active components responsible for the activities [10].

ME can significantly reduce peak blood glucose levels and insulin response levels (Kimura et al. [14]; Mudra et al. [15]), providing protection to the blood glucose metabolic function of healthy and hyperglycaemic subjects $[14,16]$. ME can reduce oxidative stress, glucose fluctuations during postprandial periods and, more generally, glucose swings triggering effects on oxidative stress. By reducing oxidative stress, ME has the potential to slow down the process of prediabetes to diabetes and diabetes to developing complications $[17,18]$. Long-term administration of ME produced a dosedependent decrease in body weight and hepatic lipid accumulation [18], stimulated skeletal muscle 5' -AMPactivated protein kinase activity acutely without changing the intracellular energy status [19], suppressed the elevation of postprandial blood glucose and cholesterol in humans [14] and exhibited potential hypoglycaemic and hypolipidaemic effects in patients with diabetes [20]. Therefore, ME may help reduce blood glucose peaks caused by the ingestion of sugary or carbohydrate-rich foods and drinks. Mulberry tea has been shown to suppress the postprandial rise of blood glucose levels after $90 \mathrm{mi}-$ nutes of its consumption in subjects with T2DM [21].

The aim of this study is to determine the effect of three doses of a proprietary water extract of mulberry leaves standardised to contain $5 \%$ DNJ (IminoNorm ${ }^{\circ}$ ) versus placebo on blood glucose and insulin responses when co-administered with $50 \mathrm{~g}$ maltodextrin in normoglycaemic healthy adults. We also aim to determine the gastrointestinal tolerability of the mulberry extract using normal $\left(250 \mathrm{mg}\right.$ IminoNorm $^{\circledR}$ containing
$12.5 \mathrm{mg}$ DNJ), half (125 mg IminoNorm ${ }^{\bullet}$ containing $6.75 \mathrm{mg}$ DNJ) and also double (500 mg IminoNorm ${ }^{\circ}$ containing $25 \mathrm{mg}$ DNJ) the normal dose. Maltodextrin is a dietary starch with a high glycaemic index, is commonly added to many foods and beverages and has been used as a test carbohydrate in similar studies [15]. We hypothesise that ME will reduce glucose excursions without causing a disproportionate increase in insulin release, and thus its ingestion may confer protection against the development of diabetes, although further trials would be required to determine its long-term efficacy.

\section{Methods/design \\ Objectives}

The primary objective is to determine the effect of three doses of mulberry extract (IminoNorm ${ }^{\circ}$ ) versus placebo on blood glucose and insulin responses when co-administered with $50 \mathrm{~g}$ maltodextrin in normoglycaemic healthy adults. We also aim to determine the gastrointestinal tolerability of the mulberry extract using normal (250 mg IminoNorm ${ }^{\circ}$ capsule containing $12.5 \mathrm{mg}$ 1-deoxynojirimycin), as well as half (125 mg IminoNorm ${ }^{\circ}$ containing $6.75 \mathrm{mg} \mathrm{DNJ})$ and double (500 mg IminoNorm $^{\bullet}$ containing $25 \mathrm{mg}$ DNJ) the normal dose. The ME (IminoNorm ${ }^{\circ}$ ) is provided by Phynova Group Ltd, UK.

\section{Hypothesis}

Our primary hypothesis is that an appropriate dose of mulberry extract co-administered with oral maltodextrin will reduce the incremental area under the curve for plasma glucose concentration over 120 minutes in normoglycaemic adults in a dose-dependent manner when compared to co-administration with placebo. Our secondary hypothesis is that mulberry extract coadministered with oral maltodextrin will not disproportionately increase the incremental area under the curve for plasma insulin concentration over 120 minutes in normoglycaemic adults compared to co-administration with placebo.

\section{Study design}

A double-blind, randomised, repeat measure, crossover design trial will be used to study the glycaemic response (GR) and insulinaemic response (IR) to three products: one reference product and three test products. Participants will act as their own controls; that is, each participant will test all four products. The trial will be conducted by the Functional Food Centre at Oxford Brookes University. Ethical approval for the study has been obtained from the University Research Ethics Committee (UREC) of Oxford Brookes University, Faculty of Health and Life Sciences, Headington Campus, Gipsy Lane, Oxford, OX3 0BP, UK. Participants will be 
given full details of the study protocol and the opportunity to ask questions. All participants will give written informed consent prior to participation.

\section{Participants}

A sample size of 40 participants will be recruited to allow for any dropouts during the study. Healthy male and female adult participants (aged 18-60 years) will be recruited for the study in order to determine if ME can have an effect on subjects without impaired glucose tolerance.

\section{Exclusion criteria}

The exclusion criteria of the MULBERRY trial are listed in Table 1.

In addition, participants will be excluded if they are unable to comply with experimental procedures or do not follow GR and IR testing safety guidelines.

\section{Suspension criteria}

All adverse events will be monitored and recorded from the time of the screening visit through 24 hours after treatment visit. Subjects may be excluded or have the study product discontinued if the investigator decides discontinuation of the study product is in

Table 1 Exclusion criteria of the MULBERRY trial

Exclusion criteria
1. Aged $<18$ or $>60$ years
2. Pregnant or lactating
3. Body mass index (BMI) $<20 \mathrm{~kg} / \mathrm{m}^{2}$ and $>30 \mathrm{~kg} / \mathrm{m}^{2}$
4. Fasting blood glucose value $>6.1 \mathrm{mmol} / \mathrm{L}$
5. Any known food allergy or intolerance including mulberry extract
6. Medical condition(s) or medication(s) known to affect glucose
regulation or appetite and/or influence digestion and absorption
of nutrients
7. Known history of diabetes mellitus (type I/II) or the use of
antihyperglycaemic drugs or insulin to treat diabetes and related
conditions
8. Use of steroids, protease inhibitors or antipsychotics (all of which
have major effects on glucose metabolism and body fat distribution)
9. Current oral hypoglycaemic use
10. Symptomatic IBS
11. History of renal or liver diseases
12. History of clotting or bleeding disorders
13. Taken antibiotics in last 3 weeks prior to screening
14. Taking daily medications or dietary supplements that are not suitable
for the study in the opinion of the PI
15. Anaemia
16. Subject to a major medical or surgical event requiring hospitalization
within the preceding 3 months
17. Current participation in another clinical study.

the best medical interest of the subject (for example, due to noncompliance, changes in medications or dietary supplements, an adverse event or serious adverse event or a change in medical status) or if the subject requests withdrawal from the study.

\section{Sample size}

A recent study in India (which is yet to be published) in 12 healthy individuals age 18-25 using $250 \mathrm{mg} \mathrm{ME}$ dose showed a reduction in the glycaemic index of maltodextrin by $58 \%$ when compared to placebo. A sample size of 30 would allow over $90 \%$ power to detect a difference of a similar size. Being more conservative and allowing for a smaller difference to be detected in the lower concentration doses, 30 participants would still allow at least $80 \%$ power to detect a difference of $25 \%$ in the incremental Area Under the Curve (iAUC). In order to account for a potential loss to follow-up, and the possibility that our sample size may be inaccurate as it is based on a small pilot sample, we will therefore recruit 40 participants.

\section{Randomisation}

Researchers recruiting the participants will be unaware of the allocation sequence (concealed allocation). Assignment of participants to a participant number will be done according to their chronological order of enrolment in the study. The allocated participant number will identify the participants and their corresponding intervention sequence. A code break for the randomisation will be kept by the Principal Investigator and will only be broken in terms of a serious adverse event.

\section{Blinding (treatment/placebo)}

Four products will be tested in this study: one placebo reference product (four capsules containing $125 \mathrm{mg}$ microcrystalline cellulose) and three test products containing different doses of mulberry extract (test product groups receive either 1,2 or 4 capsules containing $125 \mathrm{mg}$ ME, with either 3, 2 or 0 placebo capsules respectively so that participants always take 4 capsules). The study products are double blinded to both investigator and subject. Each test/reference product will be co-administered with $50 \mathrm{~g}$ maltodextrin dissolved in $250 \mathrm{~mL}$ water. Products will be produced by Phynova and delivered to the study centre labelled as 'Product variant A', 'Product variant B', 'Product variant C' and 'Product variant $\mathrm{D}$ ' with sealed envelopes labelled with participant number and the intervention sequence for the respective participant.

\section{Study period}

In each participant, the reference product and test products will be tested in random order on (four) separate 
days, with at least a two-day gap between measurements to minimise carry over effects. The study schedule is summarised in Table 2.

\section{Outcomes}

Primary outcome:

- iAUC for plasma glucose concentration over 120 minutes.

Secondary outcomes:

- iAUC for plasma insulin concentration over 120 minutes.

- Gastrointestinal tolerability - abdominal bloating may be experienced due to reduced carbohydrate absorption. Gastrointestinal symptoms will be measured via questionnaire for 24 hours following each study visit. Subjects will use a five-point scale to rate stool consistency for each bowel movement for $0-24$ hours after the study product consumption. The five-point scale includes: 1 = watery, $2=$ loose/ mushy, $3=$ soft, $4=$ formed, $5=$ hard. Frequency and intensity will be set to a $10-\mathrm{cm}$ line scale (0 representing 'Absent' for frequency and 'Usual' for intensity; 10 representing 'More than usual' for frequency and 'Severe' for intensity).

\section{Recruitment}

Participants will be recruited following local advertisements, and all participants will be given full details of the study protocol and the opportunity to ask questions. All participants will give written informed consent prior to participation and will be paid $£ 10$ per visit, on

Table 2 Summary of MULBERRY trial schedule

\begin{tabular}{|c|c|c|c|c|c|}
\hline & Screening visit & $\begin{array}{l}\text { Visit } \\
1\end{array}$ & $\begin{array}{l}\text { Visit } \\
2\end{array}$ & $\begin{array}{l}\text { Visit } \\
3\end{array}$ & $\begin{array}{l}\text { Visit } \\
4\end{array}$ \\
\hline Informed consent & $\bullet$ & & & & \\
\hline $\begin{array}{l}\text { Demographic info } \\
\text { (age, contact details etc.) }\end{array}$ & $\bullet$ & & & & \\
\hline Medical history & $\bullet$ & & & & \\
\hline Inclusion/exclusion criteria & $\bullet$ & & & & \\
\hline $\begin{array}{l}\text { Compliance check } \\
\text { (fasting, drugs etc.) }\end{array}$ & & $\bullet$ & $\bullet$ & $\bullet$ & $\bullet$ \\
\hline $\begin{array}{l}\text { Physical exam } \\
\text { (BMl, waist circ. etc.) }\end{array}$ & & $\bullet$ & $\bullet$ & $\bullet$ & $\bullet$ \\
\hline $\begin{array}{l}\text { Consume product/placebo } \\
+ \text { drink }\end{array}$ & & $\bullet$ & $\bullet$ & $\bullet$ & $\bullet$ \\
\hline $\begin{array}{l}\text { Finger-prick glucose and } \\
\text { insulin testing }\end{array}$ & & $\bullet$ & $\bullet$ & $\bullet$ & $\bullet$ \\
\hline Gl questionnaire & & $\bullet$ & $\bullet$ & $\bullet$ & $\bullet$ \\
\hline Adverse event monitoring & & $\bullet$ & $\bullet$ & $\bullet$ & $\bullet$ \\
\hline
\end{tabular}

completion of all four visits. This has been determined as an appropriate amount to cover travel costs and the time spent during each visit.

\section{Procedures/study schedule}

The study will be conducted by Good Clinical Practice (GCP) certified researchers. The reference product and test products will be administered to participants in a randomised, repeated measures design. On the day prior to a test, participants will be asked to restrict their intake of alcohol and caffeine-containing drinks and to restrict their participation in intense physical activity (for example, long periods at the gym, excessive swimming, running, aerobics). Participants will also be told not to eat or drink after 10:00 pm the night before a test, although water will be allowed in moderation. Participants will be studied in the morning after an overnight fast. Participants will consume the products at a comfortable pace, within 5 minutes. The reference product and test products will be served with $50 \mathrm{~g}$ maltodextrin dissolved in $250 \mathrm{~mL}$ water. Participants will remain sedentary during each test session and will not consume any additional food or fluid. Participants will be instructed to record stool consistency for the first bowel movement after their visit and the frequency and intensity of gastrointestinal symptoms for 0-24 hours after the study product consumption.

All anthropometric measures will be taken, using standard methods, in the morning, after an overnight fast.

- Height and weight

- BMI will be calculated using the standard formula: weight $(\mathrm{kg}) /$ height $\left(\mathrm{m}^{2}\right)$

- Waist and hip circumference

The glycaemic response method used is adapted from that described by Brouns et al. [22] and is carried out in accordance with the ISO 26642:2010 standards. Blood measurements will be taken at -5 minutes and 0 minute before consumption of the reference product/ test products and the baseline value taken as a mean of these two values. Further blood measurements will be taken at 15, 30, 45, 60, 90 and 120 minutes after starting to eat. Blood glucose will be measured using the HemoCue Glucose 201+ analyser (HemoCue ${ }^{\circ}$ Ltd). The same time points will be used for determining insulin levels. At each test time point, $300 \mu \mathrm{L}$ of capillary blood (from finger pricks) will be obtained. Finger pricks will be made using the Unistik 3 single-use lancing device (Owen Mumford, Woodstock, UK) and blood will be collected into chilled Microvette $^{\circ}$ capillary blood collection tubes treated with dipotassium EDTA (CB 300 K2E; Sarstedt Ltd., Leicester, UK). The Microvette tubes will then be centrifuged and $200 \mu \mathrm{L}$ of the supernatant plasma obtained. Insulin 
concentrations in the plasma samples will be determined by electrochemiluminescence immunoassay using an automated analyzer (Cobas ${ }^{\circ}$ E411; Roche diagnostics, Burgess Hill, UK). The Cobas ${ }^{\circ}$ system is a reliable method of plasma insulin determination. The unit of measurement is $\mu \mathrm{U} / \mathrm{mL}$. Sufficient blood is taken to enable two sets of analyses to be performed at every time point, thus greatly reducing the likelihood of missing data.

\section{Statistical analysis}

This is the first rigorous study of IM and should therefore be considered as a pilot study from a statistical perspective. We will calculate the incremental area under the curve for all four study products and compare using repeated measures ANOVA to determine whether there is a statistically significant difference in plasma glucose levels and in secondary outcome measure over the 120-minute period. All models will control for the possible confounding effects of sex and BMI. The presence/absence of gastrointestinal symptoms in the 24 hours following the study visit will be assessed using logistic regression models. We anticipate a low likelihood of missing data (as sufficient blood is taken for a second analysis to be performed if the first analysis fails), and our glucose values will be measured in real time. We anticipate monophasic or biphasic glucose and insulin curve responses and will use a second order interpolation method for missing data, provided that no other data is missing from the two neighbouring time points at either side of a missing data set and that the baseline and end data values are obtained. If the baseline or endpoints are missing or more than one data point within five neighbouring time points, this data set will be deemed unsuitable and will not be included in the analysis.

\section{Discussion}

In this paper, we have suggested the clinical trial design of a dietary supplement to reduce glucose excursions without a disproportionate increase in insulin release. This pilot study will influence further work on a larger scale RCT. In this study we aim to confirm the standard dose of IminoNorm that can be consumed with food. In addition, we aim to determine the magnitude of clinical effect and to measure the frequency and intensity of any gastrointestinal side effects. Based on the results, we will aim to design a larger RCT to investigate the protective effect of ME on the development of prediabetes and diabetes in at-risk individuals or the population as a whole. The treatment effect size may be used to determine the sample size of a larger study based on similar studies on the prevention of T2DM. As mentioned previously, ME may also have a positive effect on lipid profiles, and we may decide to include further endpoints in future larger trials. Glucose-lowering agents show ethnic variations; thus, future work should include assessment in more ethnically diverse populations.

\section{Trial status}

We have now finished recruiting and most of the participants have completed all four visits.

\section{Abbreviations}

ANOVA: analysis of variance; BMI: body mass index; DNJ: 1-deoxynojirimycin; GCP: Good Clinical Practice; GR: glycaemic response; iAUC: integral Area Under the Curve; IR: insulinaemic response; ME: mulberry extract; RCT: randomised controlled trial; T2DM: type 2 diabetes mellitus; TCM: Traditional Chinese medicine; UREC: University Research Ethics Committee.

\section{Competing interests}

The authors are funded by a Technology Strategy Board/Innovate UK grant to undertake this work with Phynova (as the applicant), and our role is solely that of researchers.

\section{Authors' contributions}

RF, AG and GL participated in the initial design of the study. BS participated in the statistical analysis. CB is the chief investigator and contributed to the study design. HL and AF participated in the final review of the protocol, the recruitment of participants and the running of the trial at Oxford Brookes. $\mathrm{ML}$ contributed to the protocol and drafted the manuscript. All authors read and approved the final version of the manuscript.

\section{Acknowledgements}

The work has been funded by a Technology Strategy Board/Innovate UK grant.

\section{Author details}

${ }^{1}$ Primary Care \& Population Sciences, Faculty of Medicine, University of Southampton, Aldermoor Health Centre, Southampton SO16 5ST, UK. ${ }^{2}$ Functional Food Centre, Oxford Brooks University, Gipsy Lane Campus, Headington, Oxford OX3 OBP, UK. 3Phynova Group Ltd, 16 Fenlock Court, Blenheim Office Park, Long Hanborough OX29 8LN, UK. ${ }^{4}$ Endocrinology and Metabolism, University of Southampton and University Hospital, Southampton, UK

Received: 30 July 2015 Accepted: 2 October 2015 Published online: 28 October 2015

\section{References}

1. TeMorenga L, Mallard M, Mann J. Dietary sugars and body weight: systematic review and meta-analyses of randomised controlled trials and cohort studies. BMJ. 2012;346:e7492. doi:10.1136/bmj.e7492.

2. National Institute for Health and Care Excellence. NICE Guideline CG43. Obesity: guidance on the prevention, identification, assessment and management of overweight and obesity in adults and children. December 2006. https://www.nice.org.uk/guidance/cg43. Accessed 15 July 2015.

3. National Institute for Health and Care Excellence. NICE Guideline PH35. Preventing Type 2 diabetes: population and community-level interventions. May 2011. http://www.nice.org.uk/guidance/ph35. Accessed 15 July 2015.

4. National Institute for Health and Care Excellence. NICE Guideline PH 38. Preventing Type 2 diabetes: risk identification and interventions for high risk individuals. July 2012. http://www.nice.org.uk/guidance/ph38. Accessed 15 July 2015.

5. InterAct Consortium, Romaguera D, Norat T, Wark PA, Vergnaud AC, Schulze $M B$, et al. Consumption of sweet beverages and type 2 diabetes incidence in European adults: results from EPIC-InterAct. Diabetologia. 2013;56(7):1520-30. doi:10.1007/s00125-013-2899-8.

6. Basu S, Yoffe P, Hills N, Lustig RH. The relationship of sugar to population-level diabetes prevalence: an econometric analysis of repeated cross-sectional data. PLoS One. 2013;8(2):e57873. doi:10.1371/journal.pone.0057873.

7. Malhotra A, Noakes T, Phinney S. It is time to bust the myth of physical inactivity and obesity: you cannot outrun a bad diet. Br J Sports Med. 2015;49(15):968-8. doi:10.1136/bjsports-2015-094911. 
8. Feinman RD, Pogozelski WK, Astrup A, Bernstein RK, Fine EJ, Westman EC, et al. Dietary carbohydrate restriction as the first approach in diabetes management: critical review and evidence base. Nutrition. 2015;31(1):1-13. doi:10.1016/..nut.2014.06.011.

9. Quality and Outcomes Framework - 2012-13. http://www.hscic.gov.uk/ catalogue/PUB12262

10. Oku T, Yamada M, Nakamura M, Sadamori N, Nakamura S. Inhibitory effects of extractives from leaves of Morusalba on human and rat small intestinal disaccharidase activity. Br J Nutr. 2006;95(5):933-8.

11. Bensky D, Gamble A. Chinese herbal medicine: materiamedica, revised edition. Seattle: Eastland Press; 1993.

12. Srivastava S, Kapoor R, Thathola A, Srivastava RP. Mulberry (Morusalba) leaves as human food: a new dimension of sericulture. Int J Food Sci Nutr. 2003;54(6):411-6.

13. Chau CF, Wu SH. The development of regulations of Chinese herbal medicines for both medicinal and food uses. Trends Food Sci Technol. 2006;17:313-23.

14. Kimura T, Nakagawa K, Kubota H, Kojima Y, Goto Y, Yamagishi K, et al. Food-grade mulberry powder enriched with 1-deoxynojirimycin suppresses the elevation of postprandial blood glucose in humans. J Agric Food Chem. 2007;55(14):5869-74.

15. Mudra M, Ercan-Fang N, Zhong L, Furne J, Levitt M. Influence of mulberry leaf extract on the blood glucose and breath hydrogen response to ingestion of $75 \mathrm{~g}$ sucrose by type 2 diabetic and control subjects. Diabetes Care. 2007;30(5):1272-4.

16. Monnier L, Mas E, Ginet C, Michael F, Villon L, Cristol JP, et al. Activation of oxidative stress by acute glucose fluctuations compared with sustained chronic hyperglycemia in patients with type 2 diabetes. JAMA. 2006:295(14):1681-7.

17. Kawamori R, Tajima N, Iwamoto Y, Kashiwagi A, Shimamoto K, Kaku K, et al. Voglibose for prevention of type 2 diabetes mellitus: a randomised, doubleblind trial in Japanese individuals with impaired glucose tolerance. Lancet. 2009;373(9675):1607-14. doi:10.1016/50140-6736(09)60222-1.

18. Wu T, Qi X, Liu Y, Guo J, Zhu R, Chen W, et al. Dietary supplementation with purified mulberry (MorusaustralisPoir) anthocyanins suppresses body weight gain in high-fat diet fed C57BL/6 mice. Food Chem. 2013;141(1):482-7. doi:10.1016/j.foodchem.2013.03.046.

19. Ma X, Iwanaka N, Masuda S, Karaike K, Egawa T, Hamada T, et al. Morusalba leaf extract stimulates 5'-AMP-activated protein kinase in isolated rat skeletal muscle. J Ethnopharmacol. 2009;122(1):54-9. doi:10.1016/j.jep.2008.11.022.

20. Andallu B, Suryakantham V, Lakshmi B, Reddy GK. Effect of mulberry (Morusindica L.) therapy on plasma and erythrocyte membrane lipids in patients with type 2 diabetes. Clin Chim Acta. 2001;314(1-2):47-53.

21. Banu S, Jabir NR, Manjunath NC, Khan MS, Ashraf GM, Kamal MA, et al. Reduction of post-prandial hyperglycemia by mulberry tea in type-2 diabetes patients. Saudi J Biol Sci. 2015;22(1):32-6. doi:10.1016/j.sjbs.2014.04.005.

22. Brouns F, Bjorck I, Frayn KN, Gibbs AL, Lang V, Slama G, et al. Glycaemic index methodology. Nutr Res Rev. 2005;18(1):145-71. doi:10.1079/NRR2005100.

\section{Submit your next manuscript to BioMed Central and take full advantage of:}

- Convenient online submission

- Thorough peer review

- No space constraints or color figure charges

- Immediate publication on acceptance

- Inclusion in PubMed, CAS, Scopus and Google Scholar

- Research which is freely available for redistribution 